\title{
Kawasaki-Erkrankung oder Hyperinflammations-Syndrom?
}

Kinderärzte aus dem von COVID-19 stark betroffenen italienischen Bergamo sahen sich im Frühling vermehrt mit Kindern mit einer Kawasaki-ähnlichen Erkrankung konfrontiert [1]. Während zwischen 2015 und 17. Februar 2017 in Bergamo 19 Patienten mit Kawasaki-Syndrom diagnostiziert wurden, zeigten zwischen 18. Februar und 20. April 2020 zehn Kinder eine Kawasaki-ähnliche Symptomatik. Dr. Ulrich Neudorf, Kinderkardiologe und -rheumatologe an der Klinik für Kinderheilkunde III, Universitätsklinikum Essen, hat an der Erstellung der deutschen Leitlinie zum Kawasaki Syndrom [2] mitgearbeitet und erklärte im Gespräch wie die italienische Publikation einzuordnen ist.

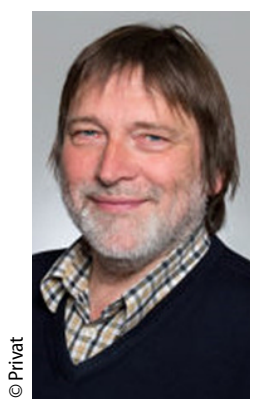

Dr. Ulrich Neudorf
Erstveröffentlichung in rheuma plus 2020 $19: 122-123$

https://

doi.org/10.1007/

s12688-020-00353-3

(c) Springer-Verlag

GmbH Austria, ein Teil von Springer Nature

2020
Was sind die aus Ihrer Sicht relevanten Beobachtungen der Lancet-Studie zum Ausbruch einer Kawasaki-ähnlichen Erkrankung in Bergamo?

Neudorf: Das Problem, das ich mit dieser Studie habe, ist, dass sie mir aus prinzipiellen Überlegungen nicht logisch erscheint. Denn formal gesehen handelt es sich beim Kawasaki-Syndrom um eine Erkrankung mit unklarer Ursache, unklarem Fieber und einer Vaskulitis mittelgroßer Gefäße. Das bedeutet, dass mit einem positiven Nachweis auf SARS-CoV-2 die Diagnose Kawasaki -Syndrom nicht zu stellen ist, da mit COVID-19 die Hypothese einer Ursache aufgestellt ist. Schon vor SARS-CoV-2 gab es Virusinfekte Hantaviren und andere - die das klinische Bild einer Kawasaki-Erkrankung zeigten.

Bei der Lancet-Studie sehen wir, dass die Kawasaki-Patienten vor COVID-19 deutlich jünger sind als die Patienten, die Kawasaki-artige Symptome während der COVID19-Infektion aufwiesen. Das Kawasaki-Syndrom betrifft im Wesentlichen Kinder unter fünf Jahren. Das Alter der mit COVID-19 assoziierten Patienten ist höher und bei innen scheinen neben mittelgroßen Gefäße auch andere von einer Entzündung betroffen zu sein.

Ich finde die Lancet-Studie interessant, aber durchaus schwierig zu bewerten. Man könnte möglicher- weise sagen, dass SARS-CoV-2 eine Erkrankung auslösen kann, die vom Erscheinungsbild her dem Kawasaki-Syndrom ähnelt und die notwendigen klinischen Kriterien erfüllt, - aber es bleiben die Fragen, ob man diese Erkrankung auch Kawasaki-Syndrom nennen und auch so behandeln sollte? Das scheint von den italienischen Kollegen so gehandhabt worden zu sein.

Es gibt auf der anderen Seite eine gemeinsame Stellungnahme der Deutschen Gesellschaft für Pädiatrische Infektiologie und der Deutschen Gesellschaft für Pädiatrische Kardiologie und Angeborene Herzfehler vom 6. Mai, in der diese Symptome "Hyperinflammationssyndrom im Zusammenhang mit COVID-19" genannt werden [3]. Diese Bezeichnung erscheint mir passender.

Beschreibt das "Hyperinflammationssyndrom im Zusammenhang mit COVID-19" dieselben Symptome, die von den Autoren der Lancet-Studie aufgegriffen wurden?

Neudorf: Die Symptome sind immer die Endstrecke einer Inflammation. Die klinischen Symptome, die das Kawasaki-Syndrom charakterisieren - also Fieber unklarer Ursache, Haut- und Schleimhautveränderungen sowie Lymphknotenschwellungen - können auch auftreten, ohne dass ein Kawasaki-
Syndrom vorliegt. Es gibt hier keine klaren Unterscheidungskriterien. Was wahrscheinlich charakteristisch ist, aber momentan nicht zu den Diagnosekriterien gehört - die auf den Kriterien der American Heart Assoziation fußen - sind Aneurysmen der Koronararterien.

Kurzgesagt: Ein klinisches Bild, das einem Kawasaki-Syndrom ähnelt, kann viele Ursachen haben. Das Ende ist immer eine schwere durch Interleukine und andere Immunmodulatoren mediierte Inflammation, die sich an den Gefäßen abspielt. Eine klare Differenzierung des Entzündungsgeschehens ist derzeit aus meiner Sicht noch nicht möglich.

In Bergamo gab es einerseits 19 Patienten mit Kawasaki-Syndrom, diagnostiziert vor 17. Februar 2020. Sie waren etwa drei Jahre alt, also im typischen Alter, in dem ein KawasakiSyndrom auftritt. Die Patienten, die während der COVID-19-Phase im Februar und April Kawasaki-ähnliche Symptome entwickelten, waren deutlich älter, im Schnitt etwa siebeneinhalb Jahre. In Relation waren das auch viel mehr Patienten. Ich glaube, was man hier in beiden Fällen sieht, ist die gemeinsame Endstrecke einer Hyperinflammation und Vaskulitis.

Aus meiner Sicht ist die LancetArbeit wichtig, weil sie beschreibt, dass es eine schwere systemische Immunantwort gibt, die wie eine Vaskulitis aussieht und die das Bild einer 
Kawasaki-Erkrankung präsentieren kann. Das ist wichtig zu wissen und mehr auch nicht. Das KawasakiSyndrom gab es auch vorher schon und es gab auch eine jahreszeitliche Häufung und Überlegungen welche viralen Infekte das Syndrom induzieren können. Aber letztendlich ist das Kawasaki-Syndrom nach wie vor eine Krankheit unklarer Ursache.

Ein Makrophagenaktivierungssyndrom gibt es übrigens auch bei verschiedenen Erkrankungen - bei genetischen Defekten, systemischen rheumatischen Erkrankungen ( $\mathrm{s} / \mathrm{A}$ ) etwa. Im Lancet-Paper ist lediglich beschrieben, dass unter SARS-CoV2-Nachweis das klinische Bild eines Kawasaki-Syndroms entstehen kann.

Ist die diagnostische Bezeichnung relevant für die Therapie?

Neudorf: Für die Therapieentscheidung ist die genaue Zuordnung möglicherweise irrelevant. Die Kawasaki-Erkrankung wird mit Immunglobulinen, Kortison und Biologika behandelt. Die Standardtherapie ist: Immunglobuline, $2 \mathrm{~g}$ pro Kilo, plus Aspirin; bei erhöhten Risikofaktoren wie Alter unter einem Jahr, männlichem Geschlecht und Herzbeteiligung würde ich zusätzlich Kortison verabreichen.

Diese Maßnahmen haben sich auch bei Hyperinflammation anderer Ursache bewährt. Als Biologika stehen uns dabei TNFa-Blocker wie das zu infundierende Infliximab zur Verfügung, außerdem die Interleukin-1 (IL1)-Blocker Kineret und Canakinumab sowie der IL-6-Blocker Tocilizumab.

Gibt es Erfahrungswerte der verschiedenen Therapie-Optionen bei Vorliegen von SARS-CoV-2?

Neudorf: Die Deutsche Gesellschaft für Pädiatrische Infektiologie sammelt gerade Daten für Deutschland. Stand der Dinge momentan ist, dass in den vergangenen Monaten zwei Fälle von Kawasaki-ähnlichen Symp- tomen erfasst wurden. Bis Anfang Mai 2020 wurden in Deutschland 128 Kinder aufgrund einer COVID19-Infektion stationär behandelt, 17 Kinder benötigten intensivmedizinische Behandlung, zwei der 128 Kinder zeigten eine Ko-Inzidenz zu Kawasaki und keines dieser Kinder ist verstorben.

Aus meiner Sicht ist der Zusammenhang der unter COVID-19 auftretenden Symptome mit dem Kawasaki-Syndrom viel Hype um wenig, der Behandlungsweg wird durch die Bezeichnung jedenfalls nicht beeinflusst.

Lässt sich etwas über die LangzeitPrognose sagen?

Neudorf: Nein, denn in Wahrheit wissen wir nicht einmal etwas über die Prognose nach einem KawasakiSyndrom. Wir tun so, als müssten wir Patienten mit Kawasaki-Syndrom nur Immunglobuline verabreichen und damit wären das Fieber weg und das Kind gesund solange die Koronararterien unbeschädigt geblieben sind.

Es gibt aber auch viele Hinweise - leider sämtlich aus Studien mit einer zu geringen Patientenzahl und einer zu differenten Methodik - darauf, dass Kinder mit KawasakiSyndrom im Verlauf ihres Lebens ein höheres Risiko für kardiovaskuläre Erkrankungen wie Vaskulopathien, koronare Herzkrankheit und Bluthochdruck haben. Um hier bessere Aussagen treffen zu können ist es in einem nächsten Schritt notwendig, alle Kinder dem gleichen Screening zu unterziehen und ihren Gesundheitszustand auch im Erwachsenenalter zu erfassen.

Haben Sie eine Empfehlung für niedergelassene Ärzte?

Neudorf: Wir haben in Deutschland gerade eine erneuerte Leitlinie zur Therapie des Kawasaki-Syndroms verabschiedet. Bei der Erstellung der Leitlinie wurde auch diskutiert, wer eigentlich das Gegenüber für Patienten mit einer KawasakiErkrankung ist. Die Kardiologen fühlen sich wegen der Herzbeteiligung zuständig - die tritt aber nur bei etwa einem Viertel der Patienten auf. Die Rheumatologen fühlen sich zuständig, aber die Patienten finden meist nicht zu den Rheumatologen. Die Patienten landen mit unklarem Fieber in der normalen Praxis.

Daher sollten praktische Ärzte und Kinderärzte einfach im Kopf behalten:Wenn etwas nicht stimmig erscheint und der Patient das klinische Bild eines Kawasaki-Syndroms zeigt, kann die Symptomatik in der COVID-19-Zeit auch auf eine Infektion mit SARS-CoV-2 zurückzuführen sein. Das sollte in einem stationären Setting auf einer Kinderklinikgetestet und überwacht werden. Eine praktische Hürde ist, dass der Umgang mit COVID-19-Tests nicht nur in jedem Land, sondern sogar in jedem Bundesland anders gehandhabt wird.

\footnotetext{
Literatur

1. L. Verdoni et al (2020) An outbreak of severe Kawasaki-like disease at the Italian epicentre of the SARS-CoV-2 epidemic: an observational cohort study. Lancet. https://doi.org/10.1016/ S0140-6736[20]31103-X

2. Leitlinie der Gesellschaft für Kinder- und Jugendrheumatologie und der Deutschen Gesellschaft für Pädiatrische Kardiologie und Angeborene Herzfehler: Kawasaki Syndrom. http://www.kinderkardiologie.org/leitlinien. Zugegriffen: 16. Jun 2020

3. Gemeinsame Stellungnahme der Deutschen Gesellschaft für Pädiatrische Infektiologie (DGPI) und der Deutschen Gesellschaft für Pädiatrische Kardiologie und Angeborene Herzfehler (DGPK) vom 6. Mai 2020: Hyperinflammationssyndrom im Zusammenhang mit COVID-19. https://dgpi.de/stellungnahmedgpi-dgpk-hyperinflammationssyndromcovid-19. Zugegriffen: 16. Jun 2020
}

Hinweis des Verlags. Der Verlag bleibt in Hinblick auf geografische Zuordnungen und Gebietsbezeichnungen in veröffentlichten Karten und Institutsadressen neutral.

Paediatr. Paedolog. 2020 · 55:232-233 https://doi.org/10.1007/s00608020-00829-7

(c) Springer-Verlag GmbH Austria, ein Teil von Springer Nature 2020 